\section{If the cap fits ...}

Telomeres - the protein-DNA complexes at chromosome ends - are protective devices that shield the DNA from degradation and endto-end fusion. They comprise a telomere-capping protein, which recognizes the telomeric DNA and regulates the telomere-forming enzyme telomerase. Such capping proteins have been characterized in ciliated protozoa, but homologues in other organisms have been elusive. Reporting in Science, however, Baumann and Cech now describe a capping protein that fits telomeric DNA throughout the eukaryotic kingdom.

The authors began with a database search in the fission yeast Schizosaccharomyces pombe, and identified an open reading frame with $40 \%$ similarity at its amino terminus to the telomere-binding $\alpha$-subunit from the hypotrichous ciliate Oxytricha nova. They then confirmed that this protein - called Pot1, for 'protection of telomeres' - binds specifically to singlestranded telomeric DNA. The affinity for DNA of a carboxy-terminally truncated Pot 1 was an order of magnitude greater than that of the fulllength protein; similar results have been

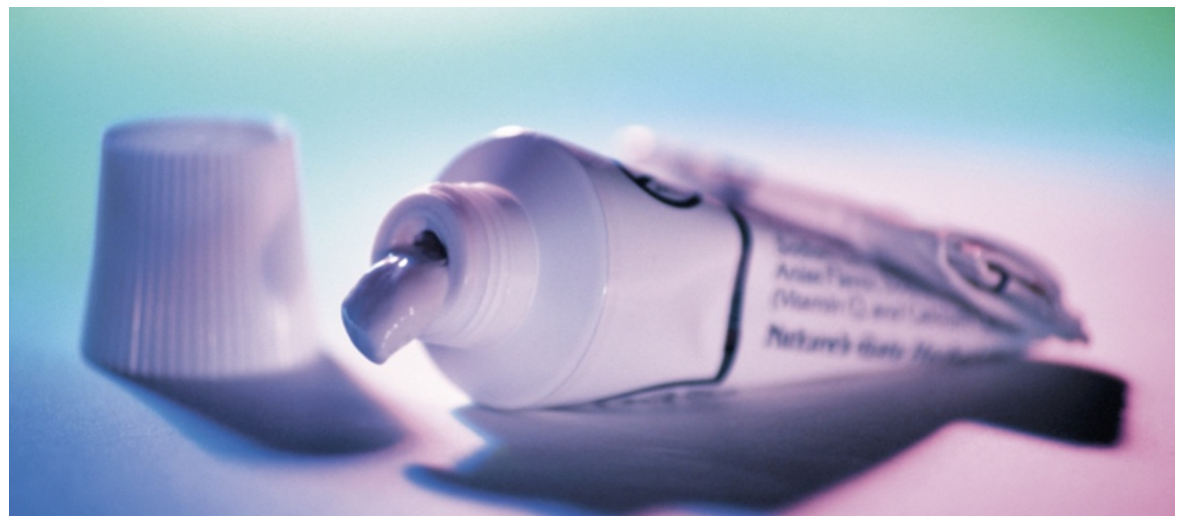

obtained with the Oxytricha $\alpha$-subunit, adding to the evidence that the two proteins are related in terms of function as well as sequence.

To investigate whether Pot1 is indeed involved in telomere maintenance, Baumann and Cech sporulated a heterozygous diploid pot $1^{+} /$pot $1^{-}$strain. The pot $1^{-}$spores formed much smaller colonies than their pot $1^{+}$sisters, and DNA staining revealed that this was due to chromosomal instability - in many pot $1^{-}$cells, the chromosomes had missegregated. Southern blotting of the DNA from pot $1^{-}$cells showed that around $5 \mathrm{~kb}$ of the telomeric DNA had been lost, supporting the idea that Pot1 protects telomeric DNA.

Could there be homologous proteins in other eukaryotes? A BLAST search with the fission yeast Pot 1 uncovered a human complementary DNA, which again showed highest sequence conservation near the amino terminus. Having cloned human POT1, the authors confirmed that the protein product binds human telomeric DNA. They then looked at the sequence specificity of the human and fission yeast proteins, and found that each protein binds much more strongly to its own telomeric DNA sequence.

Baumann and Cech detected messenger RNA for POT1 in all tissues examined, and they point out that this is consistent with POT1 being a housekeeping gene, "required to ensure the integrity of chromosome ends in all cells". They note that Pot 1 could also be involved in regulating the access of telomerase or other enzymes to the ends of chromosomes. But whatever its functions are finally found to be, the discovery of Pot 1 in fission yeast and humans suggests that the mechanism of telomere end protection is widely conserved.

Alison Mitchell

(2) References and links

ORIGINAL RESEARCH PAPER Baumann, P. \& Cech, T. R. Pot1, the putative telomere end-binding protein in fission yeast and humans. Science 292, 1171-1175 (2001)

FURTHER READING McEachern, M. J. et al. Telomeres and their control. Annu. Rev. Genet. 34, 331-358 (2000)

\title{
A brief history of the nucleus
}

Splicing does more than it was initially cut out to. As well as removing introns, splicing changes the composition of messenger RNA-protein (mRNP) complexes, which is thought to be important in communicating the nuclear history of the RNA to the cytoplasm. Y14 - a protein that binds RNA in a spliceosome-dependent manner and remains bound to mRNA in the cytoplasm — is a suitable candidate for mediating such molecular imprinting.

To determine whether Y14 binds randomly or specifically to mRNA, Narry Kim, Jeongsik Yong and co-workers microinjected radiolabelled pre-mRNAs into oocytes and, following splicing and nuclear export, separated them into nuclear and cytoplasmic fractions. By mixing these fractions with specific antisense deoxyoligonucleotides and RNase $\mathrm{H}$, the mRNA was cleaved in a sequence-specific manner. An anti-Y14 antibody was then used to show that only specific RNA fragments co-immunoprecipitated with Y14. Further analysis revealed that $\mathrm{Y} 14$ binds to a minimum region, $\sim 20$ nucleotides upstream of exon-exon boundaries, that shares no common sequence.

RNase $\mathrm{H}$ cleavage patterns were identical within nuclear and cytoplasmic fractions, indicating that $\mathrm{Y} 14$ remains bound to $\mathrm{mRNA}$ following nuclear export. Another protein, Aly/REF, also binds mRNA in the nucleus, but does not co-immunoprecipitate with mRNA in the cytoplasm. This indicates that at some stage before, during or after nuclear export, Aly/REF dissociates from mRNA, consistent with its role as a nucleocytoplasmic shuttling protein. Why, then, does $\mathrm{Y} 14$ remain bound to mRNA in the cytoplasm? A likely explanation is that it acts as a molecular imprint, indicating to the cytoplasm where the introns previously were. Beyond possible roles in nuclear export or mRNA turnover, localization or transport within the cytoplasm, a particularly attractive theory is that Y14 binding near exon-exon junctions functions in nonsense-mediated decay (NMD), a process whereby transcripts containing premature termination codons are degraded to avoid creating carboxyterminally truncated proteins. During translation of normal mRNA, Y14 (located upstream of exon-exon junctions) would be removed. As the termination codon of higher eukaryotes usually lies in the last exon of the mRNA, by the time the translating ribosome reaches it, $\mathrm{Y} 14$ proteins should have been removed. However, if a nonsense mutation occurs upstream of the final exon-exon junction, the remaining downstream $\mathrm{Y} 14$ complexes would identify these transcripts for degradation.

Katrin Bussell

(2) References and links ORIGINAL RESEARCH PAPER Narry Kim, V. et al. The Y14 protein communicates to the cytoplasm the position of exon-exon junctions. EMBO J. 20, 2062-2068 (2001) exon-exon junctions. EMBO J. 20, 2062-2068 (2001)
FURTHER READING Kataoka, N. et al. Pre-mRNA splicin imprints mRNA in the nucleus with a novel RNA-binding protein that persists in the cytoplasm. Mol. Cel/ 6, 673-682 (2000) 